\title{
Desarrollo humano y participación comunitaria: algunas reflexiones desde el enfoque gestáltico de Paul Goodman
}

\author{
Roberto Espejo*
}

\begin{abstract}
Resumen: En este artículo intentamos crear un lazo entre el enfoque gestáltico de Paul Goodman y el desarrollo humano a través del concepto de la participación comunitaria. La crítica social de Paul Goodman se conecta con las ideas centrales de la terapia gestáltica como una forma de mostrar la importancia de la participación comunitaria en una definición posible de desarrollo humano. La concepción de John Dewey de las instituciones como herramientas de desarrollo se utiliza como un punto de contacto entre las proposiciones de Goodman y el problema de la organización social. Finalmente, mostramos la importancia de considerar la educación y la responsabilidad social como una forma de evitar un punto de vista individualista del desarrollo humano.

Palabras clave: desarrollo humano, Paul Goodman, participación comunitaria, enfoque gestáltico, organización social.
\end{abstract}

\section{Human development and community participation: some ideas from Paul Goodman's gestalt approach}

\begin{abstract}
In this article we try to create a link between Paul Goodman's gestalt approach and human development through the concept of community participation. Paul Goodman's social criticism is connected to gestalt therapy's central ideas as a way to show the importance of community participation in a possible definition of human development. John Dewey's conception of institutions as tools for development is utilized as a linking point between Goodman's propositions and the problem of social organization. Finally, we show the importance of considering education and social responsibility as a way to avoid an individualistic point of view regarding human development.
\end{abstract}

Key words: Human development, Paul Goodman, community participation, gestalt approach, social organization.

Recibido: 20.05.2009

Aceptado: 01.07.2009

$* * *$

*Universidad de Paris 8, Paris, Francia. E-mail: roberto.espejo@gmail.com 
“Que ella sea política, económica, artística o religiosa, toda institución social tiene un sentido y un objetivo:

liberar y desarrollar las capacidades del individuo, sin importar su raza, su sexo, su clase o su status económico”.

(John Dewey, 1920)

\section{Introducción: en torno al desarrollo humano ${ }^{1}$}

Las palabras de John Dewey citadas más arriba (Dewey, 2003: 154) nos plantean un problema fundamental. De acuerdo a este autor el objetivo final de las instituciones que hemos creado como sociedad es el de apoyar el desarrollo del individuo. Se trata de pensar la sociedad como una estructura humanocéntrica. Desde este punto de vista cualquier entelequia (digamos una teoría económica) u organización que no vaya en esa dirección debiera ser mirada con reparo y a lo menos cuestionada. Este es una de las funciones de la filosofía según Dewey, una filosofía que debiera ser reconstruida para que pueda retomar su rol social. El pragmatismo -muchas veces mal asociado al funcionalismo y a la acción que no da pie a la reflexiónaparece entonces como una manera de responsabilizar las disciplinas científicas (ya sean en el dominio de las ciencias de la naturaleza física como en las de la naturaleza humana o social) de su acción en el mundo. Esto es cierto para las instituciones que están encargadas principalmente de esta "producción" del conocimiento, como las universidades. Desde este punto de vista, el desarrollo de las personas se transforma en uno de los fines centrales de la organización social. Pero esto no es todo. En el pensamiento de Dewey hay una condición adicional: este proceso de desarrollo humano apoyado por toda la sociedad debiera estar abierto a todos, sin importar las condiciones o características de cada uno. Esta libertad de acceso a ese desarrollo es una declinación importante de la idea de justicia. Podríamos decir que una sociedad “justa” -en relación al desarrollo humano- sería una sociedad donde todos tienen ese derecho de acceso, donde cada uno tiene un espacio para desarrollarse y para crecer $^{2}$.

Evidentemente esta idea requiere previamente una definición de lo que entendemos por desarrollo humano. De la misma manera es necesario pensar en la organización de estas instituciones y cómo puede armonizarse el fin de éstas con el fin enunciado más arriba. Y es que es más o menos claro que las instituciones pueden funcionar y prontamente "olvidar" - como si "ellas” tuvieran un ánima propia- para lo que son organizadas. Basta observar los males de la burocracia exacerbada para darse cuenta que el remedio puede tornarse peor que la enfermedad.

\footnotetext{
${ }^{1}$ El autor agradece a Claudia Drago por sus comentarios sobre este artículo.

${ }^{2}$ Evidentemente esta condición no es suficiente. Podríamos pensar también en los factores que - una vez las personas habiendo tenido acceso a esos espacios de desarrollo- tienden a hacer que los abandonen. Dichos factores no debieran depender de características como el origen social u la situación económica, si queremos pensar en una sociedad “justa”.
} 
El trabajo de los llamados “críticos sociales” es importante para mantenernos lúcidos en relación a esas instituciones y a los fenómenos sociales en general. Sin embargo, es difícil mantener una mirada crítica y lúcida (ambas características que lamentablemente no siempre vienen juntas) y al mismo tiempo guardar como centro el problema esencial que nos recuerda Dewey: es el ser humano el que está al centro y no una ideología o una forma de organización social especifica ${ }^{3}$. En Paul Goodman encontramos al mismo tiempo al crítico social y al terapeuta: un personaje que se preocupó por desarrollar la estructura social a través de su crítica pero sin olvidar que ésta era la habitación de la humanidad y no un fin en sí mismo.

Pero para volver a la idea del desarrollo humano, quisiéramos plantear como punto de partida una idea que nos parece relativamente obvia: una concepción del desarrollo humano implica a la base una cierta antropología. Evidentemente no utilizamos el término en su sentido disciplinar, sino que como un sinónimo de concepción del hombre ${ }^{4}$. Bebemos de un imaginario que nos proporciona ciertos puntos de referencia a la hora de expresarnos en torno de lo que es el ser humano y de lo que constituye su desarrollo. Existen varios puntos ligados a esta observación. En un principio, parecería que al hablar de "desarrollo" humano, tratamos con un ser humano que está inconcluso, incompleto. El trabajo de George Lapassade, por ejemplo, es bastante instructivo en relación al carácter "inacabado" del hombre. Lapassade va a cuestionar la visión del desarrollo humano como un proceso de "acabamiento", ya que ésta visión presupone la posibilidad de llegar a ese estado de "completitud". "El hombre acabado, es el hombre adulto. La perfectibilidad, con su doble significación de potencialidad y de educabilidad, supone un ideal de perfección, y por lo tanto, la posibilidad de un acabamiento" (Lapassade, 1963: 22). Pero si el hombre no es "acabable”, si el hombre está en un proceso continuo de acabamiento y de educación, ¿`cómo debemos entender el sentido del desarrollo humano? La posibilidad de este acabamiento es fundamental para comprender el sentido de cualquier "desarrollo humano" posible. Pero si reflexionamos sobre una imagen del "hombre acabado" nos encontramos más bien con una imagen que retrocede cada vez que nos acercamos a ella. ¿Debemos pensar entonces en una antropología del no acabamiento?

Esta pregunta no es anodina. Estamos lejos de querer jugar con las palabras. Si concebimos al ser humano como un ser que debe desarrollarse, ¿hasta dónde va ese desarrollo? ¿Cuáles son los límites de ese desarrollo humano? ¿Se trata de un proceso asintótico? Es decir, ¿ंen qué momento podemos decir que el ser humano ya está desarrollado? ¿Cómo cambia ese ser humano al desarrollarse? ¿Quién define que alguien es subdesarrollado

\footnotetext{
${ }^{3}$ Es necesario decir que dicha postura no es completamente "limpia” en Dewey. En muchos pasajes de sus escritos se deja entrever cómo el ser humano está sujeto a la "democracia” que Dewey identifica con el bien común. Que esto sea así implica un presupuesto filosófico y político, obviamente.

${ }^{4}$ Como sinónimo de ser humano.
} 
o superdesarrollado? ¿Bajo cuáles parámetros? Esta última pregunta abre todo un espectro de discusión. ¿Cuáles son los buenos parámetros para hablar del desarrollo humano?

Creemos que al plantear esta pregunta nos enfrentamos con un presupuesto importante del desarrollo. Cuando pensamos en este ser inacabado que debe desarrollarse podemos crearnos una imagen lineal del hombre. El hombre debe desarrollarse para ser más hombre, para ser más completo. $\mathrm{Y}$ en nuestro mundo actual este desarrollo se traduce en adquirir cosas. Ya sea cultura, títulos y diplomas, ejercicio o terapia personal. Se trata de una visión del desarrollo que es acumulativa. Las personas deben desarrollarse, luego es necesario implementar políticas que apoyen este desarrollo. Pero ¿cómo hacerlo? ¿Cómo definirlo? El conocido sistema de Maslow nos propone una pirámide de necesidades que el hombre busca satisfacer. ¿Significa que desarrollarse es sinónimo de ir escalando poco a poco en esta pirámide?

¿Pero tratamos realmente con el ser humano al considerar esta imagen lineal? Miguel Benasayag plantea por ejemplo la situación conceptual del individuo. Para él uno de los grandes problemas de los sistemas del capitalismo avanzado es el que presupone la existencia y la identidad del individuo. Podemos aprovechar su mirada y decir que en los discursos oficiales es el individuo el que se desarrolla, independiente, siempre reivindicando su derecho a ser individuo. Pero, y si -como señala Benasayag desde el psicoanálisis- ¿es verdad que el individuo no es más que un mito? “El individuo como figura central de nuestra cultura no se puede identificar de ninguna manera con un cuerpo o con la persona humana. Él es el átomo en serie que determina la base de una cultura. El individuo no es Juan, Pablo ni María, él no es ni ustedes ni yo, es una forma de organización y de dominación social” (Benasayag, 2004: 16). De esta manera, “en el neoliberalismo avanzado, el individuo no es otro que el nombre del lazo social mismo regulado por la ley de la ganancia y del interés” (Benasayag, 2004: 28). El individuo -en el sentido de Benasayag- se transforma en una construcción social perversa, funcional al sistema del capitalismo avanzado, y se inserta en las políticas que promueven el desarrollo humano como destinatario. El "individuo" usurpa al ser humano. En vez de buscar construir una sociedad más humana (a través de la búsqueda del desarrollo del hombre) construimos una sociedad más individualista (a través de la búsqueda del desarrollo del individuo).

Nos encontramos entonces frente a un problema que presenta varias aristas. De un lado tenemos que para poder considerar el "desarrollo humano” teniendo verdaderamente al "ser humano” en el centro, no podemos confundirlo con una imagen creada con el interés de mantener una cierta situación social. Es fácil, por ejemplo, decir que el ser humano necesita de comunicación para desarrollarse y que por lo tanto una compañía de servicios de Internet sirve al desarrollo humano. Bajo esa égida cualquier política que apunte a satisfacer una "necesidad" - muchas veces "creada" artificialmente - iría en la dirección del desarrollo humano. Creemos, desde nuestra humilde opinión, que no se trata de esto. 
Por otra parte, está el problema de cómo organizamos las instituciones -y el lazo de éstas con las personas- para ir en la dirección que señala Dewey. Desde este punto de vista, el desarrollo humano y el desarrollo social están íntimamente ligados. De hecho para Dewey este lazo era la definición moral de la democracia: "podemos definir la democracia de muchas formas, pero desde el punto de vista moral, la exigencia democrática consiste en juzgar las instituciones políticas y las organizaciones industriales en función de su contribución al desarrollo general de cada uno de los miembros de la sociedad” (Dewey, 2003:154).

Los seres humanos nos organizamos socialmente para poder vivir mejor. Al menos esto es lo que se nos hace creer desde una "educación cívica" humilde y un tanto anquilosada que se nos transmite en la infancia y en la juventud. Pero este "vivir mejor” encierra toda una ideología detrás. Lo que para unos puede ser una "mejor vida" para otros puede no serlo y viceversa. Hay aquí algo de una hegemonía impuesta que debiera al menos cuestionarnos un poco. Es por esto que debemos tener cuidado de mirar lo que hay detrás del “desarrollo humano” como idea.

En este artículo quisiéramos presentar y comentar una manera de entender el desarrollo humano a partir del enfoque gestáltico. Esta mirada se relaciona profundamente con la idea expuesta por Dewey de que las organizaciones debieran siempre estar subsumidas a este objetivo mayor. Esta manera de entender el desarrollo humano fue descrita por Paul Goodman en los años 60. Goodman es sobre todo conocido por su influencia en el desarrollo de la terapia gestáltica, aunque en Latinoamérica es más bien eclipsado por el trabajo de otro de sus creadores: Fritz Perls. Pero una de las características interesantes de Goodman es que él no fue sólo terapeuta: su implicación y su labor como crítico social fue muy importante en los Estados Unidos de los años 60 y 70 . Esta doble característica de este personaje lo vuelve relevante si nos interesamos en los lazos entre el desarrollo humano y la organización social, a través de la idea evocada por Dewey.

El hecho de que Goodman haya sido relegado a un nombre casi olvidado en Latinoamérica, eclipsado en el mundo de la Gestalt por Pearls, ha llevado a que sus propuestas sociales sean de la misma manera desconocidas. A pesar de esto, y especialmente en Estados Unidos, las personas que estuvieron activas en los movimientos de "contra cultura" de los años 60 recuerdan muy bien los trabajos de Goodman, sobre todo en el ámbito de la educación y de la crítica social en general.

Las ideas de Goodman aparecen dentro de un contexto bastante complejo. Podríamos decir que se trata de un intento de pensar al mismo tiempo el ser humano en su dimensión psicológica y social, a través del trabajo de alguien que fue artista, terapeuta y crítico de la sociedad. Como veremos su forma de pensar este lazo puede rastrearse a la tradición progresista americana y a otras corrientes como el anarquismo. Sin embargo, creemos que se trata de una mirada original y que puede servir de inspiración para pensar los lazos entre el desarrollo humano, la participación ciudadana y, por lo tanto, la educación en general. 


\section{Un acercamiento a Paul Goodman ${ }^{5}$}

Paul Goodman nació en 1911, en Greenwhich Village, New York, en el seno de una familia judía. Durante casi toda su vida vivió en esta ciudad, la cual amó profundamente y plasmó en su novela más importante, The Empire City (La Ciudad Imperio). Goodman creció absorbido por la atmósfera de la ciudad de Nueva York, por sus parques, museos y bibliotecas (Wall, 2003).

Hizo sus estudios primarios y secundarios en Nueva York y obtuvo una licencia en letras en el College de la misma ciudad. Siempre obtuvo las mejores calificaciones de su clase, caracterizándose por su capacidad para los idiomas. Taylor Stoher señala que Goodman había recibido la mejor educación pública gratuita del mundo (Stoher, 1998: 17). En 1931 se le puede ver trabajando como lector de manuscritos para la Metro Goldwin Meyer. Luego de obtener su licencia asistió como estudiante libre a la universidad de Columbia y de Harvard. En 1954 recibió un doctorado en literatura por la Universidad de Columbia.

Goodman trabajó en varias instituciones de educación superior como la Universidad de Chicago, la escuela Manumit de educación progresiva (Nueva York), la universidad de Wisconsin, el College Sarah Lawrence y el College experimental de la universidad de San Francisco (Vincent, 2004).

Como lo remarca Stoher (Perls, Hefferline, Goodman, 2001: 325) ${ }^{6}$ durante los años treinta las lecturas de Goodman se centraron sobre todo en los teólogos neo protestantes, al mismo tiempo que las exégesis tradicionales. En los años cuarenta los trabajos de Kirkegaard, Buber y Kafka le influenciaron profundamente. Goodman se consideraba ante todo un artista, un poeta. Se decía hombre de letras y en su trabajo teórico y de crítica siempre intentó mostrar la pertinencia y la utilidad que tenía la tradición intelectual y espiritual de occidente sobre los problemas de la sociedad actual (Vincent, 2003). Pero a pesar de su imagen de hombre intelectual occidental, Goodman exploró las tradiciones orientales donde encontró conceptos fundamentales, especialmente en la filosofía taoísta (Goodman, 1947b:12 et p.67). De la misma manera, y especialmente ligado a su posterior labor crítica en educación, Goodman recibió como inspiración las ideas del movimiento progresista en Estados Unidos, especialmente el trabajo de John Dewey (Goodman, 1960: 81).

Pero es quizás su relación con la terapia gestáltica la que lo hizo conocido fuera de los estados Unidos. Antes de encontrarse con Fritz Perls, el padre de este acercamiento terapéutico, Goodman comenzó un análisis de tipo freudiano y reicheano. De hecho conoció a Wilhelm Reich, pero decidió seguir una terapia con uno de sus alumnos, Alexander

\footnotetext{
${ }^{5}$ Para más detalles de la biografia de Goodman se puede consultar el libro de Stoher (1998).

${ }^{6}$ De ahora en adelante PHG, 2001.
} 
Lowen, que iba más tarde a hacerse conocido por haber desarrollado la "bioenergética".

En 1946, en Africa del Sur, Fritz Perls había leído un artículo escrito por Goodman sobre el trabajo de Wilhelm Reich. Perls había recibido alguna influencia de Reich en la elaboración de su propio acercamiento terapéutico y, por lo tanto, se sintió bastante intrigado por este autor americano. Llegando a estados Unidos, Perls buscó a Goodman, considerándolo como un aliado potencial. Goodman era conocido en los círculos intelectuales de Nueva York, sobre todo por su relación con las ideas anarquistas, por lo cual no le fue difícil contactarlo. Así, Goodman formó parte de un grupo de trabajo donde Perls y su esposa Laura hicieron converger una serie de disciplinas como la literatura, la investigación médica, la educación y la fotografía (PHG, 2001: 325).

Según cuenta la historia, Goodman recibió de parte de Perls un manuscrito sobre su acercamiento terapéutico el cual él debía trabajar y poner en forma. Esta misión se transformó rápidamente en una colaboración, hasta el punto de que muchos capítulos del libro que resultó eran más bien de Goodman que de Perls (PHG, 2001: 327). Se podría decir que Goodman formalizó teóricamente lo que Perls desarrollaba intuitivamente.

Con la publicación del libro (Terapia Gestáltica: novedad, excitación y desarrollo) y luego de esta colaboración, Goodman se transformó en uno de los "fundadores" de la terapia gestáltica, sobre todo en la zona de Nueva York ${ }^{7}$ y de Cleveland. Goodman comenzó a trabajar como terapeuta él mismo, hasta que un cambio en la ley le prohibió el ejercicio dado que no era psicólogo profesional.

Fue en los años 60 que Goodman comenzó a ser reconocido como crítico social. En 1960 publicó su libro más conocido (fuera de su trabajo sobre la gestalt), Growing Up Absurd, el cuál fue rechazado por 16 editoriales antes de ser publicado. En el prefacio de este libro, nuestro autor pone de manifiesto que en su texto él relaciona lo que se lee todos los días en el periódico, la "desgracia del sistema organizado" (Goodman, 1960: ix) con el descontento de la generación que está creciendo. Con este libro Goodman comienza su "carrera pública” como crítico social, donde encontró su lugar en medio de los movimientos estudiantiles de los años 60.

Pero no es en ese momento que comienza a expresar sus ideas políticas. En el "panfleto de mayo" escrito en 1945, él ya había mostrado su

\footnotetext{
${ }^{7}$ Posteriormente Perls se mudó al conocido centro de Esalen (en el sur de los estados Unidos) donde siguió su trabajo como terapeuta y formador de terapeutas. Casi nunca hizo referencia a Goodman a pesar de la colaboración que habían tenido en el pasado. A pesar de esto el nombre de Goodman figura dentro de los fundadores “oficiales” de la terapia gestáltica. Como ya fue mencionado, su contribución y su postura crítica en relación a la sociedad son prácticamente desconocidas (o bien ignoradas a propósito) en los círculos asociados a la “terapia gestáltica” en Chile.
} 
posición política, muy cercana al anarquismo. Según él, intentaba construir un mundo libre a través de vivir en el “aquí y el ahora” de forma libre (PHG, 2001: 231). Goodman se reconocía como anarquista, siguiendo específicamente la tradición de Kropotkin.

Podemos así dividir su vida en tres grandes etapas. En una primera ejerció esencialmente como escritor de novelas y de cuentos. En una segunda etapa, y tras encontrarse con Fritz Pearls, desarrolló el cuadro teórico de lo que iba a llamarse la terapia gestáltica. En la última etapa de su vida saltó a la fama por su crítica social ${ }^{8}$, específicamente por su crítica al sistema de educación en Estados Unidos con sus dos obras Growing Up Absurd (1960) y Compulsory Miseducation (1971). infarto.

Goodman murió el 2 de Agosto de 1972 en New Hampshire, de un

\section{El enfoque Gestáltico}

La terapia gestáltica fue desarrollada por Perls, Goodman y Hefferline $^{9}$, y toma su nombre del trabajo de la psicología de la gestalt (llamada también psicología de la forma). Perls y su mujer Laura tuvieron contactos con el trabajo de un grupo de psicólogos de la percepción en Berlín, donde trabajaron con el concepto central de la dinámica entre la figura y el fondo ${ }^{10}$. Este grupo estudiaba los fenómenos de la percepción sobre todo de los fenómenos visuales- en relación a la capacidad del hombre de "completar" una cierta forma (gestalt) a partir de ciertos elementos. Es decir, frente a una serie de elementos incompletos, las personas tienden a encontrar una forma posible y a "agregar" los elementos que faltan para dar coherencia a los elementos presentados en relación a la “forma” (gestalt) considerada. Esta idea fue tomada por Perls, quien la aplicó al área de la terapia y construyó a partir de ésta un método de trabajo incorporando varias otras tendencias como el trabajo corporal.

Perls fue muy influenciado por el trabajo de Kart Goldstein en Alemania, quien desarrolló su teoría de la “autorregulación organísmica” en la cual propuso que un organismo tenía la capacidad natural de regularse en relación a su medio ambiente (PHG, 2001:325) ${ }^{11}$. Por otra parte, y como lo señala Stoher, Goodman estudió también los textos de la escuela de Berlín

\footnotetext{
${ }^{8}$ Como dato interesante es importante decir que Goodman dio conferencias en el CIDOC de Ivan Illich. Illich le agradece incluso en la introducción a su libro "Deeschooling Society".

${ }^{9}$ En realidad el libro « Terapia Gestáltica » comporta dos partes, una teórica y otra de ejercicios. Goodman contribuyó en la primera parte, estando la segunda a cargo de Hefferline. ${ }^{10} \mathrm{El}$ ejemplo típico de estos fenómenos es el de la figura de la copa que dependiendo de como se observe puede transformarse en dos rostros que se miran; otro es el de la joven que se transforma en una anciana.

${ }^{11}$ Encontramos también esta idea en los trabajos de Francisco Varela y Humberto Maturana.
} 
sobre la psicología de la Gestalt, en particular los de Goldstein (PHG, 2001:325). Fue a partir de este conocimiento de las bases del acercamiento que logró construir un cuadro teórico para el método terapéutico de Perls.

Consideremos entonces brevemente la teoría de la terapia gestáltica expuesta por Goodman. Éste comienza definiendo la existencia de un "organismo" y de un "ambiente”. Estas dos estructuras son puestas en contacto a través de una frontera. Podemos pensar, por ejemplo, en el caso de la piel: a través de ésta el organismo se pone en contacto con el exterior que lo rodea. Por organismo se debe entender un ser humano, animal u incluso a una estructura social. Según Goodman "en toda investigación biológica, psicológica u sociológica, debemos partir de la interacción entre el organismo y su medio ambiente” (PHG, 2001: 50).

El estudio de un fenómeno tal nos lleva a constatar que la separación entre lo que llamamos "organismo" y lo que llamamos "ambiente" es una conceptualización. En otras palabras, podemos preguntarnos ¿dónde termina el organismo y dónde comienza el ambiente? ¿Podemos estar completamente seguros de hacer esta distinción? A partir de esta observación Goodman propone el contacto producido en la frontera de estas dos estructuras como la realidad esencial (PHG, 2001:49).

Podemos considerar, de la misma manera, el organismo en contacto e interacción con su medio ambiente como un conjunto. Goodman llamó a este conjunto el "campo organismo-ambiente". Su tesis de base era que cada vez que estudiamos un fenómeno asociado a un organismo es necesario considerar que éste es parte de un fenómeno de campo. En otras palabras, no podemos considerar los organismos como aislados de su ambiente. Pero esta relación no debe entenderse como solamente física, sino que también social. Así, "la relación entre el organismo humano y el ambiente no es solamente física, sino también social. De esta manera en todo estudio del hombre como en el caso de la fisiología, la psicología o la psicoterapia, debemos hablar de un campo en el cual interactúan al menos los factores socioculturales, animales y físicos” (PHG, 2001:51).

Pero volvamos a la importancia del contacto. Según Goodman el contacto es un ajuste. Se trata de una situación dinámica donde el organismo actúa sobre el ambiente y viceversa. El organismo busca alcanzar un equilibrio con su ambiente y de esta manera se crea una nueva configuración de campo organismo/ambiente. Podemos resumir las conclusiones de Goodman de la forma siguiente (PHG, 2001: 53):

a) Todo contacto es creativo y dinámico

b) El contacto no puede aceptar pasivamente lo nuevo

c) Lo nuevo debe ser asimilado

d) Todo contacto es un ajuste creador entre el organismo y el ambiente.

A partir de estas ideas Goodman define lo que él llama el "Self": 
"Llamamos SELF al sistema de contactos en todos los instantes. Como tal, el self varía adaptándose, sus variaciones siguen las necesidades orgánicas dominantes y la presión, los estímulos del ambiente". "El SELF es precisamente el integrador; es la unidad de síntesis como lo diría Kant” (PHG, 2001: 58). El SELF es entonces el sistema de los ajustes creadores (PHG, 2001: 74) entre el organismo y el medio ambiente. ¿Dónde está el SELF? Su función se manifiesta en la frontera de contacto. Pero esta frontera "no está aislada del ambiente, ella está en contacto con el ambiente, tiene que ver con los dos, con el ambiente y con el organismo" (PGH, 2001: 217) ${ }^{12}$.

Vemos entonces que el SELF no es una estructura fija sino que más bien un proceso continuo que yace en la frontera. Es necesario entender entonces el SELF como un proceso creador de ajuste con el ambiente. Esto implica, por ejemplo, que desde la mirada gestáltica una "neurosis" sería una incapacidad de hacer frente a los cambios en el proceso entre el organismo y su ambiente. De este punto podemos derivar la importancia que tiene para Goodman la creatividad: es a través de esta creatividad que es posible mantener este equilibrio y lograr una situación "sana". Este "self" es asociado con una percepción del organismo y de su ambiente en un momento presente. En la práctica gestáltica desarrollada por Perls y Goodman, esto se traduce en el conocido "aquí y ahora” como eje central de su método terapéutico. Así, a diferencia del psicoanálisis clásico, no se trata de buscar causas de los problemas actuales en el pasado sino que focalizarse en su manifestación en el presente.

\section{El Desarrollo Humano y la comunidad}

Desde lo expuesto más arriba resulta que el desarrollo humano puede ser entendido como un proceso donde la persona llega a estar en un equilibrio con el ambiente que lo rodea. El problema fundamental es que una vez que este equilibrio ha sido obtenido, se mantiene por muy poco tiempo. La clave de este equilibrio está entonces en las palabras "creatividad” y “dinámico”. Dado que las condiciones externas cambian continuamente, el ser humano debe buscar un ajuste creativo que vaya en movimiento con estas condiciones. La inercia es imposible y el hecho de querer llegar a un estado fijo es una ilusión. Todos lo hemos experimentado más de alguna vez al querer llegar a una situación, ya sea económica, profesional u emocional estable como sinónimo de “acabada”, una situación donde los sucesos no se alteren, donde podamos “descansar” y vivir cómodamente.

Este deseo de llegar a ese estado nos aleja -según las ideas expuestas por Pearls y por Goodman- del "aquí y del ahora”, único "instante" que tiene una existencia verdadera para nosotros. Quedarse en el pasado o vivir en una proyección continua en el futuro nos hace olvidar lo que hacemos en

\footnotetext{
${ }^{12}$ Goodman cita a menudo las ideas de Aristóteles como base de su concepto del SELF. Ver PHG, 2001: 217.
} 
un presente efímero, pero único instante real en el tiempo donde podemos actuar. Esta es la visión que está a la base de este enfoque de la psicología del ser humano, y a partir de la cual podemos pensar una antropología del hombre "inacabado". Hay un devenir continuo que busca este ajuste creador en una frontera de contacto. Pero esta frontera pone precisamente al hombre de cara a su realidad social y en particular a sus instituciones. La idea de este "self” del que hablara Goodman nos lleva a considerar la unión entre el desarrollo "humano” y la organización social que lo permite o lo impide. En el "self” la identidad del sujeto se descompone en la conciencia individual y en su relación con el ambiente. Este ambiente son los otros (una componente intersubjetiva) pero también la relación que tiene el sujeto con las instituciones que lo rodean y lo condicionan. Podemos pensar este problema como la relación entre el individuo y la comunidad de la que forma parte. El desarrollo humano, entendiendo al humano como ser inacabado que busca este ajuste creador de forma continua, se transforma en un proceso de construcción permanente ligado íntimamente con lo local.

Podemos tomar las ideas sobre el enfoque gestáltico para comprender la importancia de la idea de comunidad en Goodman. Para él, el problema estaba en el "sistema organizado" el cual dificultaba la conexión entre las personas y su propia realidad. Un sistema social debiera tener como fin último el desarrollo de los seres humanos que lo componen. La cuestión central es formulada por Goodman de la forma siguiente: "La pregunta moral no es si el hombre es “suficientemente bueno” para un tipo de organización social, sino que si esta organización social es útil para desarrollar sus potencialidades de inteligencia, de gracia y de libertad" (Goodman, 1968b:19). Reencontramos la idea del progresismo deweyeano en relación a las instituciones.

Detrás de esta imagen percibimos la idea de promover el contacto entre el organismo (los seres humanos) y el ambiente (el entorno social que los rodea). Como hemos visto, esta relación debe establecerse a través de un proceso dinámico de ajuste creador. ¿Qué significa esto en lo concreto? Nos parece que por ajuste creador deberíamos entender una forma de organización que permite la vida de la comunidad, es decir, una organización donde las personas pueden desarrollarse como tales y donde pueden actuar como seres autónomos. Para esto no es tan sólo necesario el pensar un sistema de organización, también demanda de las personas que tomen en sus manos una cierta responsabilidad en su organización. El desarrollo humano se ve íntimamente relacionado a la participación de las personas en la vida social. El problema a la base está en permitir la actividad creadora en esta “frontera” entre las personas y la organización social. Esto implica una cierta autonomía para tomar decisiones y tener la experiencia de poder modificar la realidad que los rodea. El lazo con la participación ciudadana y la democracia participativa es evidente.

Si la organización social tiene como objetivo el desarrollo del ser humano, podemos evaluar la eficacia de este sistema a partir de la medida en que éste promueva dicho objetivo. Así, por ejemplo, el sistema de edu- 
cación debiera facilitar el desarrollo de la autonomía para lograr establecer este equilibrio y ajuste creador en la base de la comunidad. Lo mismo debiera ocurrir con el sistema de gobierno. Desde este punto de vista, cada vez que observamos un sistema de organización social debemos preguntarnos dónde está su labor de facilitar o promover el desarrollo de las personas.

Roszak llama al modelo de Goodman un “comunitarismo”, diciendo que su crítica social siempre tuvo la misma finalidad: "limitar los poderes de nuestro industrialismo monstruoso para que se someta a la ética del pueblo o del barrio” (Roszak, 1970: 220). De la misma manera Vincent señala con una imagen muy vívida que "subdividir la sociedad para preservar la unidad de la persona en vez que dividir la persona para asegurar la unidad de la sociedad: tal es el proyecto general de Goodman” (Vincent, 2003b).

Se trata entonces de subsumir la organización social al desarrollo de las potencialidades del ser humano. Tomando como bandera de batalla la autonomía de las personas, estas observaciones nos llevan a mirar con desconfianza un sistema altamente centralizado, donde se dan los procesos burocráticos y el alejamiento de los tomadores de decisiones de los eventos que tienen lugar día a día en la comunidad. Por esto, y siempre desde esta filosofía del equilibrio entre el organismo y el ambiente, un sistema social descentralizado en relación al poder debiera ser la manera obvia de organizar las cosas.

Mucho se ha hablado de la descentralización, y la verdad es que es necesario tener una imagen adecuada de lo que por esto se quiere dar a entender. En el contexto de este artículo, y siempre tomando el cuadro teórico de la filosofía de la gestalt de Goodman, podemos caracterizar un sistema centralizado y uno descentralizado en base a una serie de parámetros. Un sistema centralizado se caracteriza por: a) la función a realizar es el objetivo de la organización y no el de las personas, b) las personas son consideradas como personal de la organización, c) los cargos más altos de la organización tienen más autoridad que los bajos, d) la información se compila en el nivel bajo y luego es procesada de manera que las personas que se encuentran en el nivel suprior pueden utilizarla, e) las decisiones son tomadas en el centro de toma de decisiones superior, para ser transmitidas a los niveles inferiores, f) la organización está dividida en varios departamentos de operación, a los cuales se les asigna personal con distintos roles. Por su parte, el principio de descentralización puede ser descrito de la manera siguiente: a) son las personas que están involucradas en la función de la organización. Esta es la forma de cooperación utilizada para lograr esta función, b) la autoridad es delegada lejos de la cima de la organización, de manera de tener muchos centros creadores de políticas y tomadores de decisiones, c) la información es compilada y discutida en contactos "cara a cara” entre el terreno y el centro de toma de decisiones, d) cada persona se vuelve consciente de la operación completa y trabaja para cumplirla de acuerdo a sus propias capacidades. 
En relación a esta segunda forma de pensar las organizaciones, Goodman señala que "históricamente este sistema de asociación voluntaria dio la mayoría de los valores de la civilización, pero hoy en día es considerado como completamente inviable bajo las condiciones modernas" (Goodman, 1968 b: 3-10). Esto nos lleva a la tradición de los padres fundadores de los Estados Unidos, con sus colonias y su organización altamente descentralizada. Para Goodman ese modelo tenía lecciones importantes a ser consideradas en los problemas actuales (Goodman, 1968 b: 32 - 33).

Pero la mirada de éste sobre la descentralización no es fanática. Él señala que ella no es siempre practicable. Habrá momentos en los cuales una concentración temporal de poderes es necesaria, como por ejemplo, en el caso de una emergencia. El problema se presenta cuando este tipo de organización tiende a continuar su existencia aún después que las condiciones exteriores dejaron de existir (Goodman, 1968 b: 9). De la misma forma él señala que hay funciones en la sociedad que no pueden ser descentralizadas como, por ejemplo, el control aéreo.

La organización de las instituciones sociales de una forma descentralizada, de acuerdo a las características antes mencionadas tendría como objetivo fomentar el contacto entre las personas y su realidad social. Obviamente esto no puede realizarse en todos los casos y, por lo tanto, debe considerarse la factibilidad técnica de tal sistema. El contacto fomentado por esta manera de organización sirve en sí como un espacio de desarrollo personal, ligado al desarrollo social. En una "versión débil” del sistema completamente descentralizado de Goodman, el desarrollo aparece ligado entonces a la autonomía de las personas para potenciar su propio desarrollo local.

\section{Autonomía, alienación y desarrollo humano}

¿Cómo se articula el desarrollo humano con la autonomía y el desarrollo local (comunitario)? Siguiendo el modelo antes visto, el desarrollo de las personas se entiende como el desarrollo de la capacidad creativa de adaptación a las condiciones del medio ambiente. La interrupción de esta capacidad lleva a un anquilosamiento que individualmente nos lleva a lo que se describe como una "neurosis" (en el sentido gestáltico del término). Aplicado a un nivel social esto quiere decir que las estructuras de organización debieran responder también flexiblemente a las situaciones de sus entornos, y lo que es más importante, tener los espacios para hacerlo.

El desarrollo humano se ve entonces ligado a la autonomía y a la capacidad de participación en la vida de las comunidades donde las personas viven. En realidad hay dos direcciones en las que debemos mirar: la dirección psicológica y la dirección de la acción en el mundo social. Esta última puede entenderse como participación ciudadana. Se trata del grado de compromiso que una persona puede tener al presentársele la posibilidad de hacerse responsable de un aspecto (o varios) de la situación de su comunidad. 
Así por ejemplo, Cornelius Castoriadis identifica una sociedad autónoma con una sociedad no alienada (Castoriadis, 1975: 150). Para él la autonomía es un proyecto a definir. "Es el nomos que da todo su sentido al proyecto de la autonomía. Ser autónomo, para un individuo u una colectividad no significa "hacer lo que uno desea" o lo que nos place al instante, sino que darse sus propias leyes” (Castoriadis, 1997: 198). Pero ¿en qué sentido hacemos esto? ¿Qué significa darse sus propias leyes? Como lo señalamos antes, hay dos direcciones a estudiar. Para Castoriadis se trata de atacar el problema analizando las restricciones que se nos imponen: la psicológica y la social. En efecto, Castoriadis no lo separa del cuadro psicoanalítico, considerando la idea Lacaniana de que el inconsciente "es el discurso del otro en mí mismo” (Castoriadis, 1975: 152). Tomando el problema de la libertad del hombre en relación a sus impulsos inconscientes, la autonomía (y por ende el desarrollo humano) se transforma en un problema psicosocial o de relaciones sociales (Castoriadis, 1975: 159).

Pero este cambio de relación se inscribe en un cuadro bien definido. El lado social está incluido en el individual ya que esta "voz del otro que habla en mí” proviene de un imaginario. La relación al otro (a través del imaginario) se traduce en una presencia continua de los otros frente a mis acciones y mis decisiones. Esta componente intersubjetiva nos lleva a considerar la autonomía como un problema de relaciones sociales (Castoriadis, 1975: 159).

Si entendemos entonces el problema del desarrollo humano como el problema de la autonomía, nos vemos enfrentados a un estado de alienación cada vez que esta autonomía es bloqueada. La alienación aparecería entonces ligada a la relación individuo/institución. Las instituciones se transforman en "vivas” y toman el poder sobre el ser humano. El hombre olvida que ha sido él quien las ha creado y se transforma en su esclavo. "La alienación no es inherente a la historia ni la existencia de la instituciones como tales. La alienación aparece como una modalidad de la relación a la institución, y por su intermedio, a la historia [...] La alineación se presenta primeramente como alienación de la sociedad a sus instituciones, como autonomización de las instituciones en relación a la sociedad” (Castoriadis, 1975:171). Este bloqueo de la autonomía en visto por Goodman como una pérdida de contacto entre el organismo (la persona) y su medio ambiente. Sus proposiciones en torno a la organización descentralizada tendrán, por lo tanto, el sentido de restablecer este contacto o bien de mantenerlo.

La mirada de Castoriadis nos permite ligar el tema de la alienación con el desarrollo humano. Si el desarrollo humano está ligado con la participación en la vida social y la autonomía, y si al mismo tiempo entendemos la alienación como este poder que tendrían las instituciones sobre el ser humano, debemos decir que finalmente el desarrollo humano debe entenderse como un proceso continuo de desalienación. Para esto -y volviendo a Dewey y a Goodman- las instituciones debieran ser pensadas no sólo como estructuras que permiten la organización social sino también como estructuras que promueven el desarrollo del hombre. 


\section{La educación como desarrollo humano}

Hemos presentado un modelo de desarrollo humano que está altamente ligado con la participación social. Las personas se desarrollan en la medida que participan y establecen un lazo activo son su ambiente social. La participación ciudadana se transforma entonces en un aspecto del desarrollo humano.

Pero ¿cuáles son los otros aspectos? El desarrollo de las potencialidades del individuo, como lo señala Dewey, es una condición necesaria. Pero dichas potencialidades deben ser puestas al servicio del desarrollo de la comunidad. Desde este punto de vista, el desarrollo humano lineal del que habláramos, que en principio es infinito, encuentra su lazo con la finitud. Un ser humano desarrollado no es necesariamente el que ha desarrollado todos sus aspectos potenciales, sino el que desarrollando algunas de sus capacidades, es capaz de ponerlas al servicio de su comunidad y participar en la vida social de ésta.

Este proceso reúne dos condiciones importantes: de una parte sirve para poner en contacto a la persona con su medio ambiente y así fomentar la creatividad y, por lo tanto, evitar los problemas de "pérdida de contacto" (que llevan a una neurosis según el enfoque gestáltico). Pero el dar los espacios de participación a las personas no es suficiente. Existen en cada uno resistencias y bloqueos psicológicos que pueden llevarnos a cerrar las avenidas posibles de participación. Es por ello que un acompañamiento que ayude a las personas en esta etapa de su desarrollo es necesario. No creemos que se trate forzadamente de un proceso "terapéutico" (aunque en algunos casos puede serlo) sino más bien de uno educacional. La educación se transforma entonces en un proceso dual: de un lado el desarrollo de las capacidades y potencialidades del individuo, y de otro la aplicación de éstas al entorno social. Un movimiento hacia fuera y un movimiento hacia adentro se hacen necesarios para pensar el desarrollo humano y nos muestran la importancia de incluirlo en el centro de la preocupación educacional.

Uno de los grandes problemas de la educación en nuestros días es el de considerarla como un bien de consumo más. Los estudiantes consumen educación, consumen diplomas para aumentar sus ingresos en el futuro. Este ha sido la invocación de la teoría del capital humano desde sus inicios y el fundamento teórico para la transformación del sistema de educación en nuestro país (Chile): el pasar de un sistema que piensa la educación para el hombre a un sistema que piensa la educación para el individuo; de una educación humanista a una individualista; que implica mucha preocupación por estándares académicos, como los resultados del SIMCE o de otras pruebas estandarizadas internacionales. Pero si consideramos como central el volver a dar a la educación la importante tarea de velar por el desarrollo de las personas -el desarrollo de la "cabeza, la mano y el corazón” de los que hablaran pedagogos como Pestalozzi-, ¿ es esto suficiente? 


\section{IDH, medidas cuantitativas y participación}

Como es sabido, los esfuerzos del PNUD por avanzar en la cuantificación del desarrollo humano más allá del PIB per cápita, que solo toca la esfera económica, han generado el índice de desarrollo humano (IDH) como indicador. Este índice considera -según el equipo del PNUDque tres son los factores más importantes en la vida de un ser humano: la salud y la longevidad, la educación y el nivel de vida. Para medir éstos se utilizan respectivamente como indicadores la esperanza de vida al nacer, la tasa de alfabetización de los adultos, la tasa bruta combinada de matrícula y el ingreso ${ }^{13}$ (Fongang, 2000: 91).

Salta a la vista que un tal índice está lejos de "medir" el desarrollo humano en un país, aunque es un avance importante respecto del PIB per cápita. Aparte del problema obvio de la medición de lo inconmensurable, existe el problema técnico de cómo operacionalizar ciertas variables que podrían ser medidas. Nos parece que según lo que hemos estado discutiendo, y si consideramos que la participación de las personas en su ambiente social es un factor importante del desarrollo de dichas personas, una variable que pretenda cuantificar dicha participación es factible de ser incluida y medida.

¿Cómo medir esta participación?; esto dependerá de cuáles sean las instancias reales de participación comunitaria que se dé a las personas. ¿Podría ser la tasa de participación en los procesos de presupuestos participativos? Como señala Daniel Schulgurensky, "los presupuestos participativos son una escuela de ciudadanía [...] los presupuestos participativos son un lugar donde los ciudadanos aprenden haciendo, donde adquieren una gran variedad de habilidades políticas, conocimiento, actitudes y valores, y donde llegan a ser más democráticas, tolerantes y preocupadas por los otros” (Schulgurensky, 2004: 10). Estos procesos de participación ciudadana -actualmente presentes en varias ciudades del mundo- pueden tal vez ser una pista para replantear y reformular nuestras ideas entorno a lo que el "desarrollo humano" significa.

\section{Comentarios Finales}

1) Desde el modelo presentado, el desarrollo humano tiene sentido si consideramos al ser humano como un organismo que está en un contacto permanente con su medio ambiente social. Según el enfoque gestáltico el proceso de contacto debe ser un proceso de ajuste creador que permita la evolución dinámica de la zona de contacto. Desde un punto de vista individual esto significa una relación "sana” de la persona con el medio ambiente (las otras personas y las instituciones donde se ve inmersa). Desde un punto

\footnotetext{
${ }^{13}$ En realidad es el PIB per cápita ajustado según la paridad de poderes de compra.
} 
de vista social esto significa la existencia de instituciones que tienen su razón de ser en el desarrollo de las personas.

2) La descentralización del poder parece ser la manera evidente de fomentar el contacto de las personas con su realidad social. Este tipo de descentralización no es, sin embargo, un fin en sí. Lo importante es que las personas puedan participar y no sean alienadas por las instituciones con las cuales interactúan. Existen obviamente los problemas clásicos asociados a la descentralización: la corrupción, la asimetría entre localidades producto de las capacidades distintas de las personas, la desigualdad fruto de las condiciones socioeconómicas de los habitantes de distintas zonas, la contraposición entre el desarrollo local y la integración ${ }^{14}$. Estos puntos nos muestran la necesidad de pensar en un sistema de organización que vele por el derecho de acceso que mencionábamos en la introducción de este artículo pero que, al mismo tiempo, permita la participación ciudadana y el desarrollo comunitario. Las personas tienen derecho a esgrimir el poder, siempre y cuando eso no vaya en desmedro del principio de justicia antes invocado.

3) El desarrollo humano a través del ajuste creador antes mencionado ha sido descrito como un proceso de búsqueda de equilibrio. La definición del equilibrio dinámico buscado se mantiene asociada a la idea de poder mantener un estado psicológico centrado en lo que Goodman llama el "aquí y el ahora": poner la atención en el presente y no vivir en una eterna proyección hacia al pasado u hacia el futuro. Esta vida centrada en el presente, sumada a la participación social antes descrita, se reencuentran con la idea deweyeana de la reconstrucción de las disciplinas científicas como un problema de responsabilidad social. El equilibrio interior (psicológico) se ve unido a la acción concreta sobre nuestro medio ambiente social, como expresión de una conciencia puesta en el presente y los problemas que éste nos plantea.

4) Así, el desarrollo humano no es el desarrollo del individuo de Benasayag. El desarrollo de la persona implica una componente individual pero también una componente social muy importante. No podemos considerar el desarrollo humano como el desarrollo de capacidades o competencias sin considerar los lazos que las personas establecen con sus medios sociales para ponerlas a su servicio. Mirando la filosofía gestáltica de Goodman podemos considerar una gestalt individualista como una terapia individual donde el aspecto social se deja de lado. El "desarrollo humano" visto desde esta perspectiva no es más que una forma de fomentar el indivi-

\footnotetext{
${ }^{14}$ Por ejemplo, y en relación a la multiculturalidad, en un sistema altamente descentralizado las comunidades de distintas etnias -asumiendo que éstas se concentran geográficamentetenderían a mantenerse aisladas. Lo mismo puede suceder si reemplazamos el problema de la etnia por el problema de la clase social. Nótese sin embargo que digo "mantenerse aisladas” ya que la descentralización solamente pondría sobre el tapete un problema ya presente: el de la segregación de una comunidad.
} 
dualismo, generar una ruptura entre las personas y la realidad de la sociedad y reproducir un orden inhumano. Considerar de esa manera el desarrollo humano es simplemente una re-apropiación por la parte de lo que Goodman llamaba el "sistema organizado". En el lenguaje de Dewey debiéramos decir que el desarrollo humano implica una responsabilidad social. Esta responsabilidad aparece ligada al equilibrio entre el organismo y el medio que definiera Goodman y que expresaran de otras maneras personajes como Francisco Varela. Pero el aporte de Goodman consiste en haber aplicado esta idea a la organización social y a enfatizar cómo el desarrollo de las personas estaba intrínsecamente unido a su actividad en el mundo en que vivían.

5) Creemos importante establecer un vínculo sólido entre el desarrollo humano, la educación y la responsabilidad social. Un desarrollo humano desconectado de estos otros puntos puede transformarse en un desarrollo individualista, accesible solo a unos pocos y que a la larga fomenta un modelo de sociedad donde cada uno vela por sus intereses personales. En este sentido las ideas que hemos discutido en este artículo apuntan a dar una justificación de la importancia de considerar la conexión y la participación entre las personas y sus realidades sociales cercanas como un factor esencial del desarrollo humano. Abriendo los espacios de participación adecuados la sociedad se transforma en una gran escuela, donde cada uno puede experimentar ese "aquí y ahora" que lo conecta con los otros y es de eso estamos seguros - un motor de evolución personal. 


\section{Bibliografía}

Benasayag, M. (2004, p.e. 1998), Le mythe de l'individu, La Découverte, Paris.

Castoriadis, C. (1975), L’institution Imaginaire de la Société, Seuil, Paris. Idem (1997), Faire et à Faire : Les carrefours du labyrinthe V, Seuil, Paris.

Dewey, J. (2003, p.e. 1982), Reconstruction en Philosophie, Editions Léo Scheer, Pau,

Fongang, S. (2000), L'indicateur de développement humain du PNUD, l'Harmattan, Paris.

Goodman, Paul et Percival (1947a), Communitas : means of livelihood and ways of life, Vintage, New York.

Goodman, Paul (1947b), Kafka's Prayer, Hillstone, New York.

Idem (1960), Growing Up Absurd: problems of youth in organized society, Vintage, New York.

Idem (1962), Utopian essays and practical proposals, Vintage, New York.

Idem (1968), People or Personnel and Like a Conquered Province, Vintage, New York.

Idem (1969), Five Years: thoughts during a useless time, Vintage, New York.

Idem (1970), New Reformation: Notes of a Neolithic Conservative, Vintage, New York.

Idem (1971), Compulsory Miseducation, Penguin, New York.

Idem (1973), Collected Poems, Random House, New York.

Idem (1997), La critique sociale et autres textes, Atelier de Création Libertaire, Lyon.

Idem (2001), The Empire City: A Novel of New York City, Black Sparrow Press, Santa Rosa.

Lapassade, G. (1963), L'entrée dans la vie : Essai sur l'inachèvement de l'homme, Minuit, Paris.

Perls, Frederick; Hefferline, Ralph; Goodman, Paul (2001), GestaltThérapie : nouveauté, excitation et développement, L'Exprimerie, Bordeaux. 
Roszak, Théodore (1970), Vers une contra culture, réflexions sur la société technocratique et L'opposition de la jeunesse, Éditions Stock, Paris.

Schulgurensky, D. (2004), Participatory Budget : A Tool for Democratizing Society, consultado en Enero del 2009 en : http://fcis.oise.utoronto.ca/ $\sim$ daniel_schugurensky

Stoehr, Taylor (1998), Aquí, ahora y lo que viene, Cuatro Vientos, Santiago de Chile.

Vincent, Bernard (2003), Le Virgile de l’Amérique en : Transatlantica, State of the Union, [En ligne]. Puesto en línea el 27 de Marzo del 2006, consultado el 6 de abril 2008 http://transatlantica.revues.org/ document539.html.

Idem (2003b), Présent au Monde : Paul Goodman, L’Exprimerie, Bordeaux. Idem (2004), Paul Goodman, Prophète du Présent en : Esprit, Septembre 2004, Paris.

Wall, Richard (2003), The radical individualism of Paul Goodman, consultado el 18 de abril de 2009 en : http://www.lewrockwell.com/orig3/ wall10.html 